\title{
A Radial Point Interpolation Method for Pricing Options on a Dividend Paying Asset
}

\author{
Abdelmgid O. M. Sidahmed \\ Department of Mathematics, Faculty of Science \& Arts, Rabigh \\ King AbdullAziz University, Rabigh, Saudi Arabia \\ Department of Mathematics and Applied Mathematics \\ Faculty of Mathematical Sciences \& Statistics \\ AL-Neelan University, Khartoum, Sudan \\ abdelmgid@gmail.com
}

\begin{abstract}
We present the radial point interpolation method (RPIM) to solve problems for pricing American and European put options on a dividend paying asset. Using RPIM, we get a system of ordinary differential equations which is then solved by a time integration methods . To resolve the difficulties associated with solving the free boundary problem associated with American options, we use a penalty approach. Numerical experiments are presented which prove the computational efficiency of the RPIM.
\end{abstract}

\section{Keywords}

European put options, American put options, dividend paying, radial point interpolation method

\section{INTRODUCTION}

Options on dividend paying assets are more popular than those on non-dividend paying assets.

Several attempts wear made in the past to solve these option pricing problems through a variety of techniques. We describe a few of them below.

Barone-Adesi and Whaley [1] gave simple analytic approximations for pricing exchange-traded American call and put options written on commodity futures contracts. Their approximations were computationally efficient than those obtained by binomial method or standard finite-difference methods.

Mallier and Alobaidi [11] used laplace transform methods to study the valuation of American call and put options with constant dividend yield.

Meyer [12] illustrated that a straightforward numerical implementation of the time discrete method of lines for the Black-Scholes equation can readily cope with the disappearance and reappearance of the early exercise boundary. They discussed the performance of the method by computing option prices when dividends are paid discretely at a known rate or known amount as well as with a constant dividend yield.

Kallast and Kivinukk [5] derived a method for pricing and hedging American options written on a dividend-paying asset. This method is based on Kim equations presented in [8]. They demonstrated that a simple approximation of the Kim integral equations by quadrature formulas leads to an efficient and accurate numerical procedure. This approximation was accompanied by the Newton-Raphson iteration procedure in order to compute the optimal exercise boundary at each time.

Battauz and Pratelli |2| analyzed some problems arising in the evaluation of American options when the underlying security pays discrete dividends. They studied the problem of maximizing the expected gain process over stopping times taking values in the union of disjoint, real compact sets.

Company et al. [3] obtained the numerical solution of a modified Black-Scholes equation modelling the valuation of stock options with discrete dividend payments. They used a delta-defining sequence of the involved generalized Dirac delta function and applied an approach based on the Mellin transforms.

Vellekoop and Nieuwenhuis [15] presented a method to deal with cash dividends pricing equity options, under the assumption that in between dividend dates the asset follows lognormal dynamics, and where the same dynamics are used to price all derivative products. They defined an algorithm which is computationally efficient and guarantees to generate prices that exclude arbitrage possibilities. Some other relevant works that can be worth mentioning here are those of Khaliq et al. [7] who developed adaptive $\theta$-methods for solving the Black-Scholes PDE for American options; Zhao et al. [17] who discussed some compact finite difference methods for pricing American options on a single asset with methods for dealing with optimal exercise boundary, and Tangman and Bhuruth [14] who described an improvement of Han and Wu's algorithm [4] for American options.

The RPIM has the following advantages ([9]): The shape function has the Kronecker delta property, which facilitates easy treatment of the essential boundary conditions; the moment matrix used in constructing shape functions is always invertible for irregular nodes; and the polynomials can be exactly reproduced up to desired order by polynomial augmentation. Some of these properties make the RPIM as a very powerful tool when solving complex problems like those considered in this chapter paper as well as their possible extensions to price multi-asset options. 
There are numerous variants of the mesh free approaches. One of the most popular ones is the radial point interpolation method (RPIM) we provide below a brief account of work using RPIM.

Rad et al. [13] used the meshfree radial basis point interpolation (RBPI) to solve the BlackScholes model for European and American options.They combined the RBPI with several numerical techniques, namely: an exponential change of variables, which allows them to approximate the option prices on their whole spatial domain, a mesh refinement algorithm, which turns out to be very suitable for dealing with the non-smooth options payoff, and an implicit Euler Richardson extrapolated scheme, which provides a satisfactory level of time accuracy.

Liu et al. [10] developed a meshless approach based on the Point Interpolation Method (PIM) for numerical simulation of a space fractional diffusion equation. Two fully-discrete schemes for the one-dimensional space fractional diffusion equation are obtained by using the PIM and the strong-forms of the space diffusion equation.

The rest of the paper is organized as follows. Some pricing problems for American and European options on dividend paying assets are described in Section 2 Section 3 deals with the application of radial point interpolation method to solve these problems. In Section 4 we discuss the development of the radial point interpolation method. Finally some numerical results along with a discussion on them are given in Section 5

\section{PROBLEM DESCRIPTION}

The Black-Scholes model for pricing American and European options on dividend paying assets is also an initial-boundary value problem. For European options this problem reads as

$$
\frac{\partial V}{\partial t}+\frac{1}{2} \sigma^{2} S^{2} \frac{\partial^{2} V}{\partial S^{2}}+(r-D) S \frac{\partial V}{\partial S}-r V=0,
$$

where $r$ is the risk-free interest rate, $S$ is the price of the stock, $\sigma$ is the volatility of the stock price, $D$ is the dividend yield (which is constant in the present case) on the stock, and $V(S, t)$ denotes the option's value at time $t$ for the stock price $S$.

The initial condition is given by the terminal payoff function

$$
V(S, T)= \begin{cases}\max (E-S, 0) & \text { for put } \\ \max (S-E, 0) & \text { for call }\end{cases}
$$

whereas the boundary conditions are given by

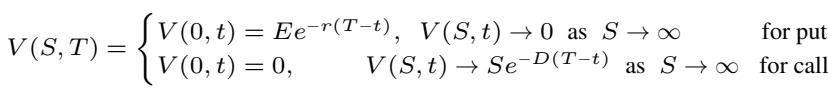

where $T$ is the maturity time and $E$ is the strike price of the option. The exact solution of the differential equation (1) with the initial condition (2) and the boundary conditions $(3)$ is given by ([16]):

$V(S, T)= \begin{cases}V(S, t)=E e^{-r(T-t)} N\left(-\widetilde{d}_{2}\right)-e^{-D(T-t)} S N\left(-\widetilde{d}_{1}\right) & \text { for put } \\ V(S, t)=e^{-D(T-t)} S N\left(\widetilde{d}_{1}\right)-E e^{-r(T-t)} N\left(\widetilde{d}_{2}\right) & \text { for call }\end{cases}$

where $N(\cdot)$ is the cumulative distribution function of the standard normal distribution with

$$
\widetilde{d}_{1}=\frac{\log (S / E)+\left(r-D+\frac{1}{2} \sigma^{2}\right)(T-t)}{\sigma \sqrt{T-t}}
$$

and

$$
\widetilde{d}_{2}=d_{1}-\sigma \sqrt{T-t}
$$

On the other hand, the American option pricing problem takes the form of a free-boundary problems. The early exercise possibility leads to the following model for the value $P(S, t)$ of an American put option to sell the underlying asset $([\overline{6}])$ :

$$
\begin{aligned}
& \frac{\partial P}{\partial t}+\frac{1}{2} \sigma^{2} S^{2} \frac{\partial^{2} P}{\partial S^{2}}+(r-D) S \frac{\partial P}{\partial S}-r P=0, \\
& \quad S>S_{f}(t), 0 \leq t<T \\
& P(S, T)=\max (E-S, 0), \quad S \geq 0 \\
& \frac{\partial P}{\partial S}\left(S_{f}, t\right)=-1, \\
& P\left(S_{f}(t), t\right)=E-S_{f}(t), \\
& \lim _{S \rightarrow \infty} P(S, t)=0, \\
& S_{f}(T)=E, \\
& P(S, t)=E-S, \quad 0 \leq S<S_{f}(t) .
\end{aligned}
$$

where $S_{f}(t)$ represents the free boundary, $E$ represent the exercise price of the option, $P$ denotes the value of the option and as before, $\sigma$ is the volatility of the underlying asset, $r$ is the risk-free interest rate, $D$ is the dividend yield on the stock.

Since early exercise is permitted, the $P$ of the option must satisfy

$$
P(S, t) \geq \max (E-S, 0), \quad S \geq 0,0 \leq t \leq T .
$$

The above problems are solved by applying the mesh free method discussed in next section.

\section{THE RADIAL POINT INTERPOLATION METHOD (RPIM)}

Following [9], we approximate the solution using the RPIM as

$$
u(x)=\sum_{i=1}^{n} R_{i}(x) a_{i}+\sum_{j=1}^{m} P_{j}(x) b_{j}=\mathbf{R}^{T}(\mathbf{x}) \mathbf{a}+\mathbf{P}^{T}(\mathbf{x}) \mathbf{b}
$$

where $R_{i}(x)$ is the $\mathrm{i}$-th radial basis function (RBF), $n$ is the number of RBFs, $m$ is the number of polynomial basis functions (PBFs), and $P_{j}(x)$ is monomial in the space coordinates $x^{T}=[x, y]$. It is clear that the conventional RBF is augmented with $m$ polynomial basis functions or in another words we can say that when $m=$ 0 , this RPIM will coincide with the conventional RBF approach. Coefficient $\mathbf{a}$ and $\mathbf{b}$ are constant vectors yet to be determined.

Coefficients $a_{i}$ and $b_{j}$ in equation (9), can be determined by enforcing equation (9) to be satisfied at $n$ nodes surrounding the point of interest $x$. This leads to $n$ linear equations, one at each node. The matrix form of these equations can be expressed as

$$
\mathbf{U}_{s}=\mathbf{R} \mathbf{a}+\mathbf{P}_{m} \mathbf{b},
$$

where the solution vector is

$$
\mathbf{U}_{s}=\left[\begin{array}{llll}
u_{1} & u_{2} & \cdots & u_{n}
\end{array}\right]^{T},
$$

the moment matrix of RBFs is

$$
\mathbf{R}=\left[\begin{array}{llll}
R_{1}\left(r_{1}\right) & R_{2}\left(r_{1}\right) & \cdots & R_{n}\left(r_{1}\right) \\
R_{1}\left(r_{2}\right) & R_{2}\left(r_{2}\right) & \cdots & R_{n}\left(r_{2}\right) \\
\vdots & \vdots & & \vdots \\
R_{1}\left(r_{n}\right) & R_{2}\left(r_{n}\right) & \cdots & R_{n}\left(r_{n}\right)
\end{array}\right]_{(n \times n)}
$$

with

$$
r_{k}=\sqrt{\left(x_{k}-x_{i}\right)^{2}+\left(y_{k}-y_{i}\right)^{2}},
$$


and the polynomial moment matrix is

$$
\mathbf{P}_{m}=\left[\begin{array}{lllll}
1 & x_{1} & y_{1} & \cdots & P_{m}\left(x_{1}\right) \\
1 & x_{2} & y_{2} & \cdots & P_{m}\left(x_{2}\right) \\
\vdots & \vdots & \vdots & & \vdots \\
1 & x_{n} & y_{n} & \cdots & P_{m}\left(x_{n}\right)
\end{array}\right]_{(n \times m)}
$$

The coefficient vector multiplying to RBFs in 9 is

$$
\mathbf{a}=\left[\begin{array}{llll}
a_{1} & a_{2} & \cdots & a_{n}
\end{array}\right]^{T},
$$

and the coefficient vector multiplying to PBFs in 9 is

$$
\mathbf{b}=\left[\begin{array}{llll}
b_{1} & b_{2} & \cdots & b_{m}
\end{array}\right]^{T} .
$$

There are $n+m$ variables in equation (10). The additional $m$ equations comes from the following $m$ constraints

$$
\sum_{i=1}^{n} P_{j}\left(x_{i}\right) a_{i}=\mathbf{P}_{m}^{T} \mathbf{a}=0, \quad j=1,2, \cdots, m .
$$

Combining equations 10 and 17 we obtain the following system of equations

$$
\widehat{\mathbf{U}}_{s}=\left[\begin{array}{l}
\mathbf{U}_{s} \\
\mathbf{0}
\end{array}\right]=\left[\begin{array}{ll}
\mathbf{R} & \mathbf{P}_{m} \\
\mathbf{P}_{m}^{T} & \mathbf{0}
\end{array}\right]\left[\begin{array}{l}
\mathbf{a} \\
\mathbf{b}
\end{array}\right]=\mathbf{G} \widetilde{\mathbf{a}}
$$

where

$$
\widetilde{\mathbf{a}}=\left[\begin{array}{llllllll}
a_{1} & a_{2} & \cdots & a_{n} & b_{1} & b_{2} & \cdots & b_{m}
\end{array}\right]^{T},
$$

and

$$
\widehat{\mathbf{U}}_{s}=\left[\begin{array}{llllllll}
u_{1} & u_{2} & \cdots & u_{n} & 0 & 0 & \cdots & 0
\end{array}\right]^{T} .
$$

Because the matrix $\mathbf{R}$ is symmetric, it is therefore clear from the structure of the matrix $\mathbf{G}$ that it will also be symmetric.

Solving system (18), we obtain

$$
\widetilde{\mathbf{a}}=\left[\begin{array}{l}
\mathbf{a} \\
\mathbf{b}
\end{array}\right]=\mathbf{G}^{-1} \widehat{\mathbf{U}}_{s} .
$$

Now equation (9) can be written as

$$
\begin{aligned}
& u(x)=\mathbf{R}^{T}(x) \mathbf{a}+\mathbf{P}^{T}(x) \mathbf{b}=\left[\begin{array}{ll}
\mathbf{R}^{T}(x) & \mathbf{P}^{T}(x)
\end{array}\left[\begin{array}{l}
\mathbf{a} \\
\mathbf{b}
\end{array}\right]\right. \\
& =\left[\begin{array}{lll}
\mathbf{R}^{T}(x) & \mathbf{P}^{T}(x)
\end{array} G^{-1} \widehat{\mathbf{U}}_{s} \quad \text { using } 21\right. \\
& =\widehat{\Phi}^{T}(x) \widehat{\mathbf{U}}_{s},
\end{aligned}
$$

where the RPIM shape functions can be expressed as

$$
\begin{aligned}
& \widehat{\Phi}^{T}(x)=\left[\begin{array}{ll}
\mathbf{R}^{T}(x) & \mathbf{P}^{T}(x)
\end{array}\right] G^{-1} \\
& =\left[\begin{array}{l}
\phi_{1}(x) \phi_{2}(x) \cdots \phi_{n}(x) \phi_{n+1}(x) \cdots \phi_{n+m}(x)
\end{array}\right]
\end{aligned}
$$

Finally, the RPIM shape functions corresponding to the nodal displacements vector $\Phi(x)$ are obtained as

$$
\Phi(x)=\left[\begin{array}{llll}
\phi_{1}(x) & \phi_{2}(x) & \cdots & \phi_{n}(x)
\end{array}\right]
$$

where

$$
\Phi_{k}(x)=\sum_{i=1}^{n} R_{i}(x) \bar{G}_{i, k}+\sum_{j=1}^{m} P_{j}(x) \bar{G}_{n+j, k}, \quad k=1,2, \cdots, n,
$$

in which $\bar{G}_{i, k}$ is the $(i, k)^{t h}$ element of matrix $G^{-1}$.

Equation (22) can be re-written as

$$
u(x)=\Phi(x) \mathbf{U}_{s}=\sum_{i=1}^{n} \phi_{i} u_{i} .
$$

The derivatives of $u(x)$ are obtained as

$$
u_{, x}(x)=\Phi_{, x}^{T}(x) \mathbf{U}_{s} .
$$

In the above, a comma indicates a partial differentiation with respect to the spatial coordinate $x$.

Equation 27] gives

$$
\frac{\partial \Phi_{k}}{\partial x}=\sum_{i=1}^{n} \frac{\partial R_{i}}{\partial x} \bar{G}_{i, k}+\sum_{j=1}^{m} \frac{\partial P_{j}}{\partial x} \bar{G}_{n+j, k}
$$

and

$$
\frac{\partial^{2} \Phi_{k}}{\partial x^{2}}=\sum_{i=1}^{n} \frac{\partial^{2} R_{i}}{\partial x^{2}} \bar{G}_{i, k}+\sum_{j=1}^{m} \frac{\partial^{2} P_{j}}{\partial x^{2}} \bar{G}_{n+j, k} .
$$

In case of Multiquadric radial basis function

$$
R\left(\left\|S-x_{j}\right\|\right)=\sqrt{\left(\left\|S-x_{j}\right\|\right)^{2}+c^{2}},
$$

the partial derivatives are obtained as

$$
\frac{\partial R\left(\left\|S-x_{j}\right\|\right)}{\partial S}=\frac{\left(\left\|S-x_{j}\right\|\right)}{\sqrt{\left(\left\|S-x_{j}\right\|\right)^{2}+c^{2}}}
$$

and

$$
\frac{\partial^{2} R\left(\left\|S-x_{j}\right\|\right)}{\partial S^{2}}=\frac{c^{2}}{\left(\left(\left\|S-x_{j}\right\|\right)^{2}+c^{2}\right)^{3 / 2}} .
$$

In the next section we will discuss the use of above RPIM in pricing European and American put options on a dividend paying asset.

\section{APPLICATION OF RADIAL POINT INTERPOLATION METHOD IN PRICING OPTIONS}

\subsection{Pricing European options on a dividend paying asset}

We approximate the unknown function $V$ (the value of the European option) using the radial basis functions as

$$
V(S, t) \approx \sum_{j=1}^{N} a_{j}(t) \phi\left(\left\|S-x_{j}\right\|\right)
$$

where $a_{j}^{\prime} s$ are unknown coefficients and $\phi\left(\left\|S-x_{j}\right\|\right)$ are the RBFs We will use the following radial basis functions for this problem

$$
\phi(S)=e^{-\left\|S-x_{j}\right\|^{2} / c^{2}},
$$

where $c$ is a positive parameter

Collocating at the same $N$ points $\left\{x_{j}\right\}_{j=1}^{N}$, equation $\sqrt{1}$ becomes

$\frac{\partial V\left(x_{i}, t\right)}{\partial t}+\frac{1}{2} \sigma^{2} S_{i}^{2} \frac{\partial^{2} V\left(x_{i}, t\right)}{\partial S^{2}}+(r-D) S_{i} \frac{\partial V\left(x_{i}, t\right)}{\partial S}-r V\left(x_{i}, t\right)=0$.

Differentiating (33), we get

$$
\begin{gathered}
\frac{\partial V\left(x_{i}, t\right)}{\partial t}=\sum_{j=1}^{N} \frac{d a_{j}(t)}{d t} \phi\left(\left\|S-x_{j}\right\|\right), \\
\frac{\partial V\left(x_{i}, t\right)}{\partial S}=\sum_{j=1}^{N} a_{j} \frac{\partial \phi\left(\left\|S-x_{j}\right\|\right)}{\partial S},
\end{gathered}
$$


and

$$
\frac{\partial^{2} V\left(x_{i}, t\right)}{\partial S^{2}}=\sum_{j=1}^{N} a_{j} \frac{\partial^{2} \phi\left(\left\|S-x_{j}\right\|\right)}{\partial S^{2}} .
$$

In case of Gaussian basis functions, we have

$$
\frac{\partial \phi\left(\left\|S-x_{j}\right\|\right)}{\partial S}=-\frac{2\left(S-x_{j}\right)}{c^{2}} e^{-\left\|S-x_{j}\right\|^{2} / c^{2}}
$$

and

$$
\frac{\partial^{2} \phi\left(\left\|S-x_{j}\right\|\right)}{\partial S^{2}}=\frac{4\left(S-x_{j}\right)^{2}-2 c^{2}}{c^{4}} e^{-\left\|S-x_{j}\right\|^{2} / c^{2}} .
$$

Substituting the expressions for various partial derivatives from equations 36- 38 into 35, we obtain

$$
\begin{aligned}
& \sum_{j=1}^{N} \frac{d}{d t}\left(a_{j}(t)\right) \phi\left(\left\|x_{i}-x_{j}\right\|\right) \\
& +\frac{1}{2} \sigma^{2} x_{i}^{2} \sum_{j=1}^{N} a_{j}(t)\left[\frac{4\left(x_{i}-x_{j}\right)^{2}-2 c^{2}}{c^{4}} \phi\left(\left\|x_{i}-x_{j}\right\|\right)\right] \\
& +(r-D) x_{i} \sum_{j=1}^{N} a_{j}(t)\left[\frac{-2\left(x_{i}-x_{j}\right)}{c^{2}} \phi\left(\left\|x_{i}-x_{j}\right\|\right)\right] \\
& -r \sum_{j=1}^{N} a_{j}(t) \phi\left(\left\|x_{i}-x_{j}\right\|\right)=0 .
\end{aligned}
$$

We can write equation (41) in form of a system of differential equations as

$$
\Phi \frac{d \mathbf{a}}{d t}+R \mathbf{a}=0
$$

where

$$
\Phi_{i j}=e^{-\left\|x_{i}-x_{j}\right\|^{2} / c^{2}}
$$

and

$$
\begin{aligned}
R_{i j} & =\frac{1}{2} \sigma^{2} x_{i}^{2}\left(\frac{4\left(x_{i}-x_{j}\right)^{2}-2 c^{2}}{c^{4}}\right) \Phi_{i j} \\
& +(r-D) x_{i}\left(\frac{-2\left(x_{i}-x_{j}\right)}{c^{2}}\right) \Phi_{i j}-r \Phi_{i j} .
\end{aligned}
$$

To solve the system described by (42), we use a $\theta$-method

$$
\Phi \frac{a^{n+1}-a^{n}}{\Delta t}+\theta R a^{n+1}+(1-\theta) R a^{n}=0,
$$

with the initial condition given by the first part of equation (2) and boundary conditions given by the first part of equation (3).

We can rewrite equation 45 as

$$
\begin{gathered}
{[\Phi-(1-\theta) \Delta t R] a^{n}=[\Phi+\theta \Delta t R] a^{n+1},} \\
a^{n}=[\Phi-(1-\theta) \Delta t R]^{-1}[\Phi+\theta \Delta t R] a^{n+1} .
\end{gathered}
$$

Equation 33 applied at all collocation point can be written in the matrix form as

$$
V=\Phi \mathbf{a} .
$$

Using equation (48), equation (47) can be written as

$$
V^{n}=\Phi^{-1}[\Phi-(1-\theta) \Delta t R]^{-1}[\Phi+\theta \Delta t R] \Phi V^{n+1} .
$$

The above equation is solved along with 2 and the first part of equation (3) to obtain the numerical solution. Also the form of this equation should be read in context to the computing process because in the problems like those considered in this paper, we usually have a final boundary value problem rather than an initial boundary value problem. To this end, note that the scheme given by (46) corresponding to $\theta=0,0.5$, and 1 are the implicit Euler, Crank-Nicolson and explicit Euler methods, respectively.

\subsection{Pricing American options on a dividend paying asset}

To solve the American option problem (7), which is a free boundary problem, we approximate the model by adding a penalty term. This leads to a nonlinear partial differential equation on a fixed domain. We consider the initial-boundary value problem

$\frac{\partial P_{\epsilon}}{\partial t}+\frac{1}{2} \sigma^{2} S^{2} \frac{\partial^{2} P_{\epsilon}}{\partial S^{2}}+(r-D) S \frac{\partial P_{\epsilon}}{\partial S}-r P_{\epsilon}+\frac{\epsilon C}{P_{\epsilon}+\epsilon-q(S)}=0$,

with the initial condition as the first part of equation (2), and the boundary conditions as

$$
P_{\epsilon}(0, t)=E, \quad \lim _{S \rightarrow \infty} P_{\epsilon}(S, t)=0,
$$

where $C \geq r E, q(S)=E-S$, and $0<\epsilon \ll 1$.

By inserting equations (33), (36)- (40) into equation (50), we obtain

$$
\begin{aligned}
& \sum_{j=1}^{N} \frac{d}{d t}\left(a_{j}(t)\right) \phi\left(\left\|x_{i}-x_{j}\right\|\right) \\
& +\frac{1}{2} \sigma^{2} x_{i}^{2} \sum_{j=1}^{N} a_{j}(t)\left[\frac{4\left(x_{i}-x_{j}\right)^{2}-2 c^{2}}{c^{4}} \phi\left(\left\|x_{i}-x_{j}\right\|\right)\right] \\
& +(r-D) x_{i} \sum_{j=1}^{N} a_{j}(t)\left[\frac{-2\left(x_{i}-x_{j}\right)}{c^{2}} \phi\left(\left\|x_{i}-x_{j}\right\|\right)\right] \\
& -r \sum_{j=1}^{N} a_{j}(t) \phi\left(\left\|x_{i}-x_{j}\right\|\right) \\
& +\frac{\epsilon C}{\sum_{j=1}^{N} a_{j}(t) \phi\left(\left\|x_{i}-x_{j}\right\|\right)+\epsilon-q(S)}=0 .
\end{aligned}
$$

We write equation (52) in form of a system of differential equations as

$$
\Phi \frac{d \mathbf{a}}{d t}+R \mathbf{a}+Q(\mathbf{a})=0,
$$

where

$$
\begin{gathered}
\Phi_{i j}=e^{-\left\|x_{i}-x_{j}\right\|^{2} / c^{2}}, \\
Q_{i}(\mathbf{a})=\frac{\epsilon C}{\Phi_{i} \mathbf{a}+\epsilon-q\left(x_{i}\right)}, \quad i=1, \cdots, N
\end{gathered}
$$

and

$$
\begin{aligned}
& R_{i j}=\frac{1}{2} \sigma^{2} x_{i}^{2}\left(\frac{4\left(x_{i}-x_{j}\right)^{2}-2 c^{2}}{c^{4}}\right) \Phi_{i j} \\
& +(r-D) x_{i}\left(\frac{-2\left(x_{i}-x_{j}\right)}{c^{2}}\right) \Phi_{i j}-r \Phi_{i j} .
\end{aligned}
$$


The $\theta$-method for equation (53) reads

$\Phi \frac{a^{n+1}-a^{n}}{\Delta t}+\theta R a^{n+1}+(1-\theta) R a^{n}+\theta Q\left(a^{n+1}\right)+(1-\theta) Q\left(a^{n}\right)=0$.

By replacing $a^{n}$ in the penalty term by $a^{n+1}$, the linearly implicit scheme for (57) is given by

$$
\Phi \frac{a^{n+1}-a^{n}}{\Delta t}+\theta R a^{n+1}+(1-\theta) R a^{n}+Q\left(a^{n+1}\right)=0,
$$

with the initial condition given by the first part of equation (2) and boundary conditions given by equation 51 .

Also note that the scheme corresponding to $\theta=0,0.5$, and 1 are the implicit Euler, Crank-Nicolson and explicit Euler methods, respectively.

\section{NUMERICAL RESULTS AND DISCUSSION}

The parameters used for the simulations for European put option on a dividend paying asset using the multiquadratic radial point interpolation method are: $r=0.05, \sigma=0.2, D=0.04, E=$ $10, t_{0}=0, T=0.5, S_{0}=0$ and $S_{\max }=30$. We have set the parameter $c$ in the radial basis function as $2 h$ where $h=\left(S_{\max }-S_{0}\right) /(N-1)$. Using the RPIM approach, the resulting problems for European put option on a dividend paying asset are presented in Table 5. The first column in this table represents values of the asset price $S$, the second column represents the exact solution, the third column indicated the numerical values of the European put option on a dividend paying asset that we obtain using the RPIM and the last column represents the error.

Table 1. Values of European put option on a dividend paying asset using radial point interpolation method

\begin{tabular}{|c|c|c|c|}
\hline $\mathrm{S}$ & Exact & RPIM(100) & Error \\
\hline 2 & 7.7927 & 7.7927 & 0.0000 \\
\hline 4 & 5.8323 & 5.8323 & 0.0000 \\
\hline 6 & 3.8720 & 3.8720 & 0.0000 \\
\hline 7 & 2.8941 & 2.8941 & 0.0000 \\
\hline 8 & 1.9443 & 1.9451 & 0.0007 \\
\hline 9 & 1.1154 & 1.1163 & 0.0008 \\
\hline 10 & 0.5271 & 0.5250 & 0.0021 \\
\hline 11 & 0.2037 & 0.2037 & 0.0000 \\
\hline 12 & 0.0653 & 0.0653 & 0.0000 \\
\hline 13 & 0.0176 & 0.0178 & 0.0003 \\
\hline 14 & 0.0043 & 0.0042 & 0.0001 \\
\hline 15 & 0.0009 & 0.0009 & 0.0000 \\
\hline 16 & 0.0002 & 0.0002 & 0.0000 \\
\hline
\end{tabular}

Table 5 presented the discrete maximum norm error and mean square norm error which calculated using

$$
\text { Error } M a x=\max _{i=1,2, \ldots, N}\left(V_{\text {exact }}-V_{\text {RPIM }}\right)
$$

and the mean square norm:

$$
R M S E r r o r=\frac{1}{N+1} \sqrt{\sum_{i=0}^{N}\left(V_{\text {exact }}-V_{\text {RPIM }}\right)^{2}}
$$

Using the RPIM approach, the resulting problems for European put option on a dividend paying asset are solved via Crank-Nicolson's
Table 2. The maximum norm error and mean square norm error

\begin{tabular}{|c|c|c|}
\hline $\mathrm{N}$ & Max Error & RMS Error \\
\hline 25 & $3.67 \times 10^{-2}$ & $1.50 \times 10^{-3}$ \\
\hline 50 & $1.40 \times 10^{-2}$ & $3.82 \times 10^{-4}$ \\
\hline 75 & $6.30 \times 10^{-3}$ & $1.16 \times 10^{-4}$ \\
\hline 100 & $2.10 \times 10^{-3}$ & $2.36 \times 10^{-5}$ \\
\hline 150 & $1.60 \times 10^{-3}$ & $1.64 \times 10^{-5}$ \\
\hline 200 & $8.81 \times 10^{-4}$ & $6.06 \times 10^{-6}$ \\
\hline
\end{tabular}

Table 3. Values of American put option on a dividend paying asset using radial point interpolation method

\begin{tabular}{|c|c|c|c|}
\hline S & RPIM21 & RPIM41 & RPIM101 \\
\hline 0.6 & 0.4014 & 0.4014 & 0.4014 \\
\hline 0.7 & 0.3019 & 0.3019 & 0.3019 \\
\hline 0.8 & 0.2049 & 0.2050 & 0.2050 \\
\hline 0.9 & 0.1216 & 0.1219 & 0.1220 \\
\hline 1.0 & 0.0646 & 0.0651 & 0.0653 \\
\hline 1.1 & 0.0321 & 0.0326 & 0.0327 \\
\hline 1.2 & 0.0156 & 0.0159 & 0.0160 \\
\hline 1.3 & 0.0078 & 0.0080 & 0.0081 \\
\hline 1.4 & 0.0042 & 0.0043 & 0.0044 \\
\hline RMS & $5.3283 \times 10^{-5}$ & $5.5987 \times 10^{-6}$ & $3.2560 \times 10^{-7}$ \\
\hline
\end{tabular}

method (i.e., $\theta=0.5$ ). Results are presented in Table 5 and Figure 1.

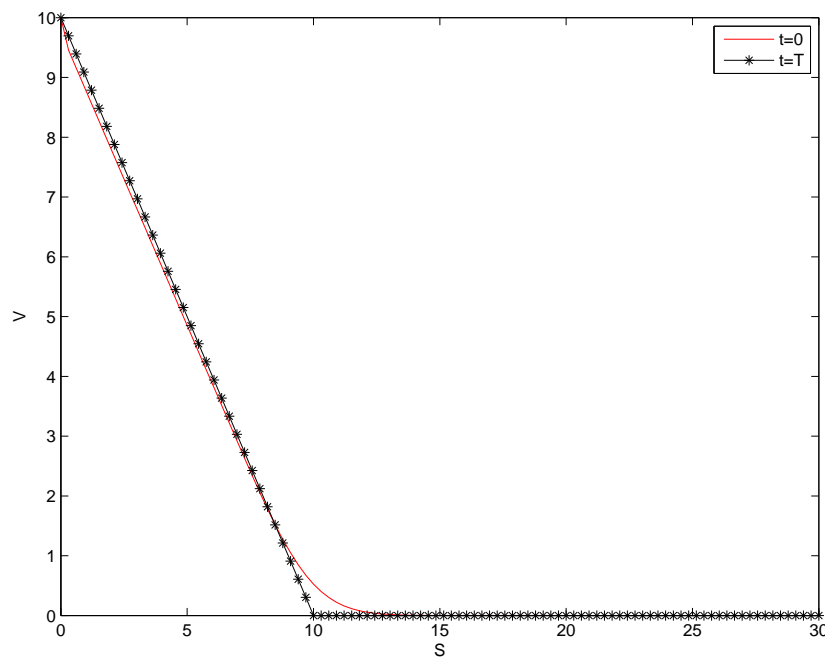

Fig. 1. Values of the European put option on a dividend paying asset using the multiquadratic radial point interpolation method are with $r=$ $0.05, \sigma=0.2, D=0.04, E=10, t_{0}=0, T=0.5, S_{0}=$ 0 and $S_{\max }=30$..

For the American put options, we choose $r=0.1, \sigma=0.2, D=$ $02, E=1, t_{0}=0, T=1, \epsilon=0.01, S_{0}=0$, and $S_{\max }=2$. We again use the Crank-Nicolson method with $\Delta t=0.01$. Using the multiquadratic radial point interpolation method, we obtain reasonably accurate results this can be seen from Table 5 and Figure 2. 


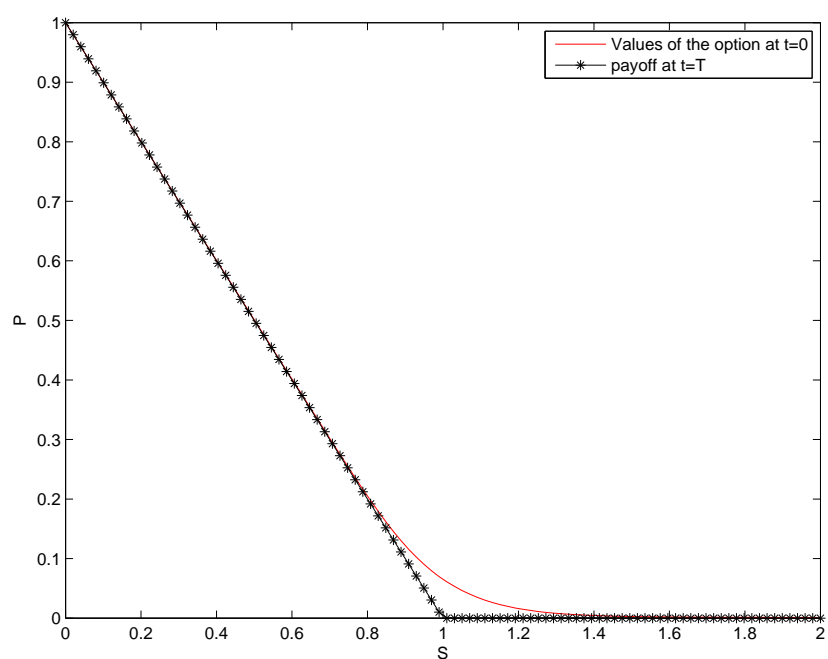

Fig. 2. Values of the Amercan put option on a dividend paying asset using the multiquadratic radial point interpolation method are with $r=$ $0.1, \sigma=0.2, D=0.02, E=1, t_{0}=0, T=1, \epsilon=0.01, S_{0}=$ 0 , and $S_{\max }=2$.

Note that in the case of the American option the exact value of $\mathrm{V}$ is not available. Therefore, we use a very accurate approximation of it with a very large number of centers and time steps (namely we set $N=300$ and $M=1000$ ).

\section{REFERENCES}

[1] Barone-Adesi G. and Whaley E. 1987. Efficient analytic approximation of American option values. The Journal of Finance, XLII (2).

[2] Battauz A. and Pratelli M. 2004. Optimal stopping and American options with discrete dividends and exogenous risk, Insurance: Mathematics and Economics, 35, 255-265.

[3] Company R., Gonzalez A.L. and Jodar L. 2006. Numerical solution of modified Black-Scholes equation pricing stock options with discrete dividend, Mathematical and Computer Modelling, 44, 1058-1068.

[4] Han H. and Wu X. 2003. A fast numerical method for the Black-Scholes equation of American options, SIAM Journal of Numerical Analysis, 41(6), 2081-2095.

[5] Kallast S. and Kivinukk A. 2003. Pricing and Hedging American Options Using Approximations by Kim Integral Equations, European Finance Review, 7 , 361-383.

[6] Khaliq A.Q.M., Voss D.A. and Kazmi S.H.K. 2006. A linearly implicit predictor-corrector scheme for pricing American options using a penalty method approach, Journal of Banking \& Finance, 30, 489-502.

[7] Khaliq A.Q.M., Voss D.A. and Kazmi S.H.K. 2008. Adaptive $\theta$-methods for pricing American options, Journal of Computational and Applied Mathematics, 222, 210-227.

[8] Kim I.K. 1990. The analytical valuation of American options, Review of Financial Studies, 3, 547-572.

[9] Liu G.R. and Gu Y. T. 2005. An Introduction to Meshfree Methods and Their Programming, Springer.
[10] Liu Q., Liu F., Gu Y.T., Zhuang P., Chen J. and Turner I. 2015. A meshless method based on Point Interpolation Method (PIM) for the space fractional diffusion equation, Applied Mathematics and Computation, 256, 930-938.

[11] Mallier R. and Alobaidi G. 2000. Laplace transforms and American options, Applied Mathematical Finance, 7, 241256.

[12] Meyer G.H. 2002. Numerical investigation of early exercise in American puts with discrete dividends, Journal of Computational Finance, 5(2), 37-53.

[13] Rad J.A., Parand K. and Ballestra L.V. 2015. Pricing European and American options by radial basis point interpolation, Applied Mathematics and Computation, 251, 363-377.

[14] Tangman D.Y., Gopaul A. and Bhuruth M. 2008. A fast highorder finite difference algorithm for pricing American options, Journal of Computational and Applied Mathematics, 222(1), 17-29.

[15] Vellekoop M.H. and Nieuwenhuis J.W. 2006. Efficient pricing of derivatives on assets with discrete dividends, Applied Mathematical Finance, 13(3), 265-284.

[16] Wilmott P., Howison S. and Dewynne J. 1995. The Mathematics of Financial Derivatives: A Student Introduction, Cambridge University Press, Oxford, UK.

[17] Zhao J. , Davison M. and Corless R.M. 2007. Compact finite difference method for American option pricing, Journal of Computational and Applied Mathematics, 206, 306 - 321. 